\title{
Elegance of fundamental laws of physics - outreach potential
}

\author{
Ivan Melo ${ }^{a, *}$ \\ ${ }^{a}$ Dept. of Physics, University of Žilina \\ Univerzitná 1, Žilina, Slovakia \\ E-mail: melo@fyzika.uniza.sk
}

From Kepler to Einstein, Dirac and Weinberg (to name a few), most physicists believed that the ultimate explanation of the Universe must possess beauty. For some of them, the beauty of the physical laws was as important as the truth. Over the last few years, however, we hear more dissenting voices fuelled by the no new physics results at the LHC, which claim that we have been led astray by aesthetics. These claims range from constructive criticism to more or less open scepticism. This situation calls for an assessment. I will argue that beauty in physics, besides being an important epistemic tool, offers a wonderful message that has an outreach potential. The topic was inspired by the discussions within the IPPOG collaboration.

40th International Conference on High Energy physics - ICHEP2020

July 28 - August 6, 2020

Prague, Czech Republic (virtual meeting)

*I gratefully acknowledge financial support by the Ministry of Education of the Slovak Republic via project FEPO. I would like to thank members of the IPPOG Collaboration for many useful discussions of the topic. 


\section{Introduction}

We were blessed with two major discoveries in fundamental physics in the last decade: the Higgs boson in 2012 and gravitational waves in 2015. There were also scores of other important results from particle physics and cosmology which stand witness to the richness and the productivity of the field. However, our hopes for new physics at the Large Hadron Collider (LHC) have not yet been fulfilled and we have to cope with this situation. Nima Arkani-Hamed said for CERN Courier that some people in the scientific community are disappointed that we have only discovered the Higgs and nothing else [1]. This could be no more than a natural psychological reaction. Physicists as human beings can be impatient. The LHC will continue to yield enormous amounts of new data in an unprecedented energy region for many years and experimentalists are enthusiastically studying the data from all possible directions.

But still, some physicists think that we may have put too much emphasis on beauty in our theories which extend the Standard model of particle physics (SM). By beauty we often mean properties such as unification, symmetry, simplicity and naturalness. They have been very successful up to the SM but, according to critics, this may not continue beyond the SM. For example, Sarah Demers said that maybe the universe is a little bit ugly [2] and Marcelo Gleiser adds that perhaps the search of perfect symmetry is not a physics idea, but a bias [2]. Ben Allanach suggests that the centuries-long quest for top-down (from theory to data) unification has stalled and calls for a more modest, bottom-up approach (from data to theory) [3]. Sabine Hosenfelder is also a sceptic. She wrote a book Lost in Math with a subtitle How beauty leads physics astray, in which she questions beauty principles, in particular naturalness [4].

Of course, it is legitimate to voice these concerns. We should be wary of the biases and the universe may not be perfectly symmetric at the fundamental level. We should not, however, forget what we have so far learned about aesthetic principles in particle physics. The fundamental laws of nature are beautiful and this is a positive message which we have not yet effectively communicated to other fields and the public.

According to a philosopher of science Jesus Bonilla, quantum physicists and mathematicians, form a special group among scientists, which he calls Platonists [5]. They believe that beauty has an important role in scientific research. The other group, Sceptics, is populated by all other scientists and philosophers of science who think that the research process is or should be independent of beauty. Clearly, as particle physicists, we are in a unique position. We have a first-hand experience with what a poet would call the splendour of this world, the splendour which remains hidden to a large degree from a layman's view.

\section{Plato and Platonists}

We will briefly describe the teaching of Plato. In his seminal work Republic, he argues that beauty is an Idea or a Form and it comes in many different forms arranged in a hierarchical order [6]. At the top we find the highest form, beauty in itself, which is perfect, eternal and without changes. Below the top there are other forms which are copies of the highest form, however, they are less and less perfect. At the bottom, we find the beauty of material objects and phenomena which shares the least amount of the perfect beauty. Plato wrote that the goal of 
education was to take the student from the bottom level as high as possible, in an ideal case to the knowledge of the perfect beauty. To see the perfect beauty means to know the full truth.

We can find many proponents of this view among leading scientists in physics. Here I offer just a small sample. Henry Poincare said "The Scientist does not study nature because it is useful to do so. He studies it because he takes pleasure in it, and he takes pleasure in it because it is beautiful" [7]. Hermann Weyl is quoted by Chandrasekhar in the following way: "In my work, I have always tried to unite the true with the beautiful; but when I had to choose one or the other, I usually chose the beautiful" [7]. And Paul Dirac tops it all by claiming "it is more important to have beauty in one's equations than to have them fit experiment" [8]. We can recognize that beauty is a great source of motivation for these founders of the field and they are not afraid of showing it. They value it as much as truth, if not more.

Frank Wilczek seems to claim that beauty is a pointer to the truth: "Having tasted beauty at the heart of the world, we hunger for more. In this quest, I think, there is no more promising guide than beauty itself" [9].

Steven Weinberg associates aesthetic properties with truth and knowledge in his horse breeder parable: when the breeder says 'That's a beautiful horse', he is not, according to Weinberg, expressing just an aesthetic emotion but the breeder knows that that is the kind of horse that wins races [10]. It appears that Plato would wholeheartedly agree with Weinberg. It is one thing to admire the horse's colour or mane (the lowest form of beauty) and another to recognize the truth and the real beauty behind the outer appearance (the potential to win races).

Finally, Arkani-Hamed sees the laws of physics as perfect, simple and also inevitable since it is hard to modify them [11].

What these and many other Platonists have in mind when they refer to beauty criteria, has different forms, typically simplicity, symmetry, inevitability and conformity with the whole $[7,12,13,14]$. My preference is symmetry and productivity, the two criteria advocated by Frank Wilczek [13]. By productivity he means a property of a theory which, starting from a few very deep principles, inevitably predicts the behaviour of many different phenomena. Productivity then also implies unity and is connected to simplicity and inevitability but unlike them, productivity seems to be better defined simply by counting and comparing the number of principles versus the number of predictions. Symmetry is also a well-defined concept as we know from Group theory.

Fundamental physics naturally fits Plato's hierarchy of forms. The highest level, which we have not reached yet, could be occupied by the hypothetical Theory of everything, characterized by the perfect symmetry and unity of the physical laws. The next level below would be taken by a theory beyond the SM, such as supersymmetry, which breaks the perfect symmetry via the spontaneous symmetry breaking. Another level below we find other theories including the Standard model and so on until we get through a series of symmetry breakings to the lowest level represented by the material objects and phenomena wherein the original perfect symmetry is mostly hidden.

\section{McAllister's theory of scientific revolutions}

There is an open problem in philosophy as to the nature of beauty. Is it an objective or a subjective property? In Platonism, beauty is considered as intrinsic to objects and hence it 
follows that by observing the object, the two scientists or philosophers would observe the same amount of beauty. A different view holds it that beauty is a value projected by an observer on to the object during observation of an aesthetic property. Different observers tend to project different amounts of beauty in response to how they view the importance or future promise of the property. This is more in line with the traditional view that beauty is in the eye of the beholder.

McAllister's theory of scientific revolutions [15], based on the concept of beauty as a value, is important for us since it explicitly describes the (possibly negative) role of beauty criteria during a scientific revolution. According to the theory, scientists use both empirical and aesthetic criteria in the process of theory evaluation. In the normal period of science, the scientists notice that certain aesthetic properties, such as symmetry, appear in empirically successful theories and the credit of these properties naturally rises over time. When facing a choice between two new theories which both passed empirical criteria, scientists choose the one with the (by now) familiar aesthetic properties. A theory with a new aesthetic property can be accepted only if it fares empirically better than theories with established aesthetic properties. The process is not easy. A part of the community is so attached to the old aesthetic properties that they continue to oppose the new line of theories despite their superior empirical performance. The community is split between conservatives and progressives, the former arguing that the new theories are ugly, the latter saying that old beauty principles no longer apply. The revolutionary period is underway and it is finished when the conservatives finally step back under the weight of experiments in favour of the new theories. The credit of the old aesthetic properties collapses and the influence of the new ones starts building up. Obviously, beauty in McAllister's theory can lead physics astray.

Platonists would, however, argue that intrinsic beauty does not disappear in the revolutionary act. For example, the power and beauty of gauge symmetry will always be appreciated by a true physicist, even if the gauge symmetry goes out of fashion. Also, the unification of electricity and magnetism in Maxwell's theory will look like an amazing feat to him. Or, for that matter, the inevitability which leads one from general covariance to general relativity. As Arkani-Hamed puts it, "Even the provisional understanding of the truth we have at any given time has a large degree of what you would call local perfection to it" and it will not disappear when we know more [11].

I suggest that there is both subjective and objective (intrinsic) part of the beauty in our fundamental theories. The former may evaporate during a revolution, the latter is eternal.

\section{Conclusions}

We have seen an interesting correspondence between Plato's hierarchy of beauty forms and the hierarchy of fundamental theories in physics. The top level of Plato's scheme is taken by perfect beauty, associated with the full knowledge and representing the full unity of all different beauty forms. The highest level of our theories could be taken by the unified theory of everything, possibly exhibiting the perfect symmetry. The lower level theories have parts of the original symmetry, with the missing parts being spontaneously broken. At the lowest level, the original perfect symmetry is hidden. All that remains are bits and pieces of some symmetry and 
a lot of asymmetries. The true, intrinsic beauty being out of sight, observers are left with the subjective beauty of appearances and conclude that beauty is in the eye of the beholder.

Physics has walked a long way from the lowest, apparent form of beauty, uncovering the staggering beauty of fundamental laws. And it found the connection between order and chaos, between symmetry and asymmetry in the form of spontaneous symmetry breaking. And this is our message for society, our outreach potential. Art, in my opinion, treads a similar path, searching for different forms of beauty, playing with the tension between symmetry and asymmetry and yes, also looking for perfect beauty.

The message of fundamental laws of physics is that of unity, symmetry, stability and the richness of forms which inevitably follows. All assets much needed in this modern world.

\section{References}

[1] Nima Arkani-Hamed, In it for the long haul (interview by Matthew Chalmers), CERN Courier 2019 (March/April) p. 45-49, https: / / cerncourier.com/a/in-it-for-the-long-haul/

[2] Sarah Scoles, What does beauty have to do with physics? NOVA 2016 (May 31), https://www.pbs.org/wgbh/nova/article/beauty-in-physics/

[3] Ben Allanach, Going nowhere fast, Aeon 2018 (June 19), https : / / aeon. co/essays / hasthe-quest-for-top-down-unification-of-physics-stalled

[4] Sabine Hossenfelder, Lost in Math, Basic Books, Hachette Book Group, 1290 Avenue of the Americas, New York, NY 10104.

[5] Jesus Zamora Bonilla, Science and the search of beauty, Mapping Ignorance 2015 (Jan 28), https://mappingignorance.org/2015/01/28/science-search-beauty-1/

[6] Plato, The Republic, 370 B.C., Penguin Books 2003, translated by Desmond Lee.

[7] Subrahmanyan Chandrasekhar, Beauty and the Quest for Beauty in Science, talk at the Fermilab symposium Aesthetics and Science 1979 (April).

[8] Paul Dirac, The Evolution of the Physicist's Picture of Nature, Scientific American 1963 (May), republished on June 25, 2010.

[9] Frank Wilczek, A beautiful question: finding nature's deep design, New York: Penguin Press, p. 9.

[10] Steven Weinberg, Viewpoints on String Theory: Steven Weinberg, NOVA 2003 (July), www.pbs.org/wgbh/nova/elegant/view-weinberg.html

[11] Nima Arkani-Hamed, The Morality of Fundamental Physics, talk at Cornell University, April 28, 2016, https://www. cornell.edu/video/nima-arkani-hamed-moralityfundamental-physics

[12] Thrinh Xuan Thuan, Chaos and Harmony: Perspectives on Scientific Revolutions of the Twentieth Century, New York, Oxford University Press.

[13] Steve Paulson, Beauty is Physics' Secret Weapon, Frank Wilczek in an interview by Steve Paulson for Nautilus 2016 (Jan 14), http://nautil.us/issue/32/space/beauty-is-physics-secret-weapon

[14] Anthony Zee, Fearful symmetry: the search for beauty in modern physics, New York: Macmillan.

[15] James W. McAllister, Beauty \& Revolution in Science, Cornell University Press 1996. 